\title{
PQLI Engineering Controls and Automation Strategy
}

\author{
Ray Bolton $\cdot$ Stephen Tyler
}

Published online: 17 May 2008

(C) International Society for Pharmaceutical Engineering 2008

\begin{abstract}
This paper discusses the development of engineering controls and automation strategy required to practically implement a Control Strategy within a Quality by Design environment. It describes the relationship to the ISPE PQLI Control Strategy model, and covers operating philosophy, record keeping, data management, and alarm strategy. Engineering and automation controls may include measurement technologies for equipment parameters (offline, at-line, in-line or on-line), univariate or multivariate process models and control models, engineering or plant procedural controls and automation systems. Concepts from ANSI/ISA S95 standards are applied.
\end{abstract}

Keywords PQLI · Design space · Control strategy $\cdot$ Critical quality attribute $\cdot$ Critical process parameter $\cdot$ Knowledge space $\cdot$ PAT Product and process knowledge $\cdot$ Process model $\cdot$ Control model

Contributing Team Members: Bruce Davis - AstraZeneca, Tom Garcia - Pfizer, Eric Ahuja - Merck, Metter Bryder - Lundbeck, Michael Hahn - Lundbeck, Gordon Muirhead - GSK, Sue Busse Lilly, Line Lundsberg - NNE Pharmaplan, Graham Cook - Wyeth, Hedinn Valthorsson - Novartis

\section{R. Bolton}

AstraZeneca,

Hazelwood House, Tytherington Business Park,

Tytherington, SK10 4XR, UK

e-mail: ray.bolton@astrazeneca.com

S. Tyler

Abbott Laboratories,

1401 Sheridan Rd.,

North Chicago, IL 60064, USA

e-mail: stephen.tyler@abbott.com

\section{Introduction}

This paper covers the development of engineering controls and automation strategy and how they can be applied in the context of the proposed ISPE PQLI Control Strategy Model as described in PQLI Control Strategy Model and Concepts [6] in this volume of the Journal of Pharmaceutical Innovation and should be read in conjunction with that paper.

The Control Strategy Model aims to facilitate communication and understanding of the concept and provide a framework for a structured approach to the development and implementation of a Control Strategy.

The model seeks to link the attributes of the product that are important to the patient, to the controls in the manufacturing process that are needed to deliver those attributes, and also to show in parallel the business requirements. The model shows three levels showing links from the finished product CQAs and other objectives through the manufacturing operations to the controls by which these are achieved.

At Control Strategy Level 1 the Critical Quality Attributes (CQAs) and other requirements are identified. Level 2 considers the critical process parameters, material attributes and components involved in meeting the CQA requirements. Level 3 covers the actual analytical, automation, and other controls of the Level 2 identified parameters and attributes. This paper focuses on the development of engineering and automation controls at Level 3.

The engineering control and automation strategy describes how the process will be controlled and documents the principles of verification to ensure that the product meets both patient critical and business requirements, and that the process has been operated in control within the approved Design Space.

The process model defines what the process requirements are whereas the control model is concerned with how 
these requirements are achieved. The control model manages the dynamics of process changes in real time.

Note that the terms Control Strategy Levels 1, Level 2, and Level 3 as used in the PQLI Control Strategy Model are different to the ISA levels referred to later in this paper.

This should be regarded as work in progress and comment and input from industry, regulators, and suppliers is welcomed.

\section{Analytical, Engineering, and Other Control Methods}

Consideration of analytical, engineering and automation controls can begin when the Level 2 strategies are well developed. The CQAs and any associated critical and noncritical process parameters will have been identified and a manufacturing route established. The production data required to assure that the product meets all regulatory, safety, efficacy, and GMP quality requirements should have been identified.

Other key performance requirements that relate to business needs such as minimising costs, maximising yields, etc while operating within the Design Space should also have been identified.

The Level 3 Control Strategy for engineering and automation controls defines how the Level 2 requirements will be implemented. Examples include measurement technologies for equipment parameters (off-line, at-line, in-line or on-line), univariate or multivariate process models and control models, engineering or plant procedural controls, and automation systems.

\section{Operating Philosophy}

The approach taken will depend on the firm's approach to lifecycle management for the particular product, e.g. whether the product is to be accommodated in an existing manufacturing plant possibly requiring modifications, or a new plant is to be designed and built. If the product is to be manufactured in a new plant, this gives an opportunity to develop or revise a high level operating philosophy. Considerations may include:

- How will the risks identified at Level 1 and Level 2 be mitigated?

- How will the production workflow be managed ${ }^{1}$ ?

- How will manufacture of the product be integrated with other systems such as Manufacturing Execution Sys-

\footnotetext{
${ }^{1}$ Options include using a traditional paper batch sheet, or alternatively a computer based electronic batch workflow system incorporating electronic signatures (in which case relevant regulations such as 21 CFR 11 must be considered)
}

tems, Materials Management Systems, or Production Scheduling Systems?

- How will the Critical Process Parameters (CPPs) be maintained in control within the process Design Space during the manufacturing process?

- How will the production data be collected and monitored to verify that all CQAs are achieved for the product?

- How will the site quality system ensure that the product meets its specifications to ensure product safety, efficacy and quality?

\section{ISA-95}

The ISA-95 international standards for the integration of enterprise and control systems [1-4] are a useful tool when establishing an operating philosophy. ISA-95 consists of a framework (Fig. 1) and terminology.

These can be used to determine which information has to be exchanged between systems for sales, finance and logistics and systems for production, maintenance and quality assurance.

The ISA S95 Function diagram describes the activities that must take place in order to produce a product. They represent the business processes required for manufacturing to take place. Activities would typically occur from left to right in Fig. 2 and the boxes from top to bottom shows the levels of integration between levels.

The ISA S95 framework can be used to define a high level operating philosophy and to summarise and document how each activity will be implemented. Some activities may be manual processes supported by Standard Operating Procedures (SOPs), while others may be implemented using commercial software for engineering control and automation.

The operating philosophy for drug substance (DS) and drug product (DP) may be different. For example, the operating philosophy for drug substance manufacture may be to have a central control room with computer based supervision and control with high levels of automation. The operating philosophy for drug product may be to have independent but automated process stages with progression from stage to stage by means of a paper based batch sheet or a Manufacturing Execution System.

If a product (DS or DP) has been developed using enhanced product development and Quality by Design (QbD) principles then product, process, science and chemical engineering knowledge may be applied to produce process models for key process stages.

These process models define the relationship between process variables and may include heat and mass balances. The objective is to predict, model and control the CPPs for each stage, their acceptable working ranges, and how they influence product CQAs. 


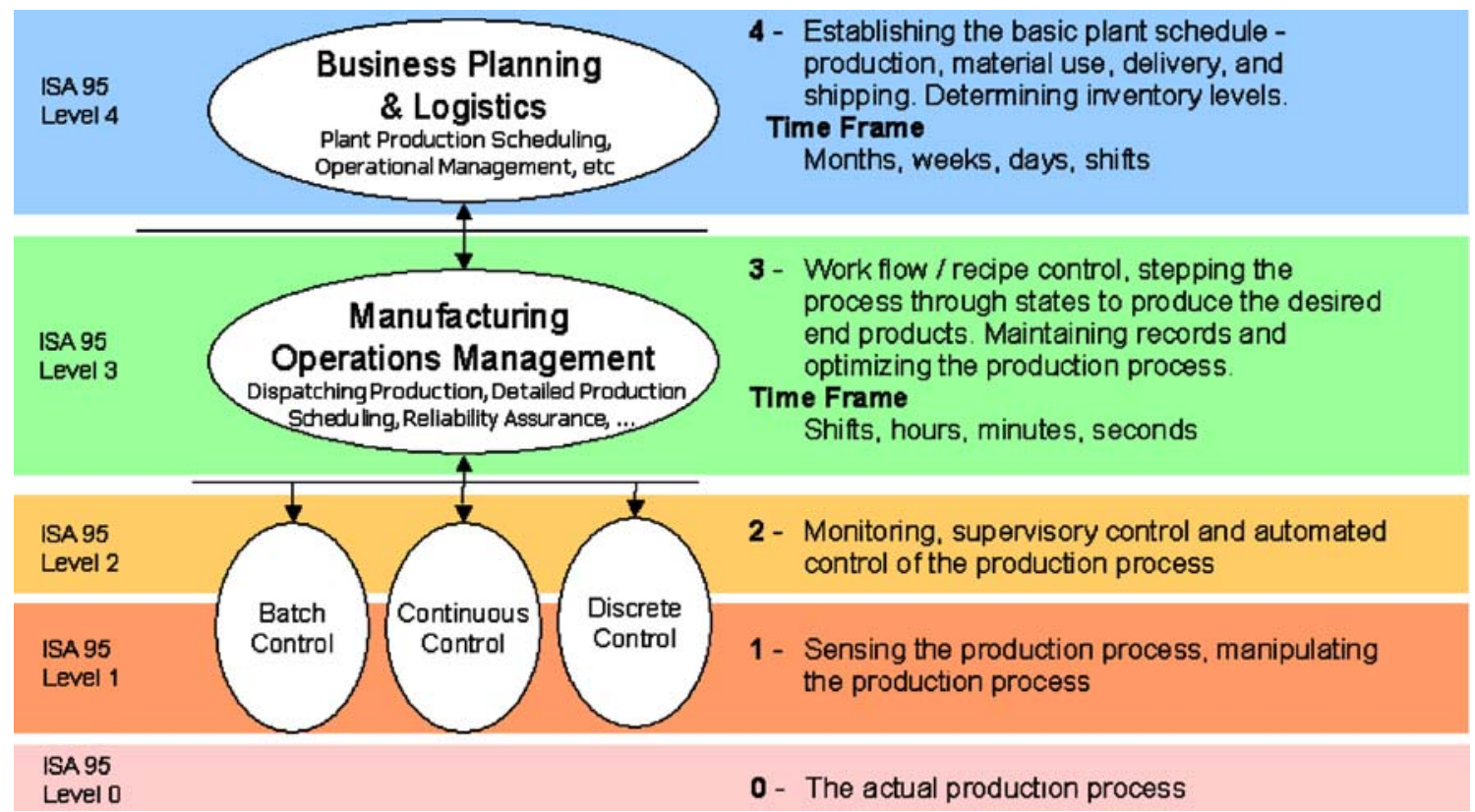

Fig. 1 The ISA 95 operating philosophy framework for manufacturing (note ISA 95 levels are different from PQLI Control Strategy Levels)

\section{Control Models}

Control models can then be developed that are designed to maintain CPPs and other process variables under control within the Design Space, corresponding to product related acceptable working limits. In a multi-product plant these process variable acceptable working limits may vary between products. The control models are concerned with the time dependant dynamics of maintaining process variables within acceptable limits, i.e. controlled within the approved Design Space.

Options available include the following:

- Use traditional recipes and implement changes within the Design Space with a streamlined validation approach. This may include focusing only on the affected variables, limiting validation to changes that are the edge of the Design Space, or using less than three runs

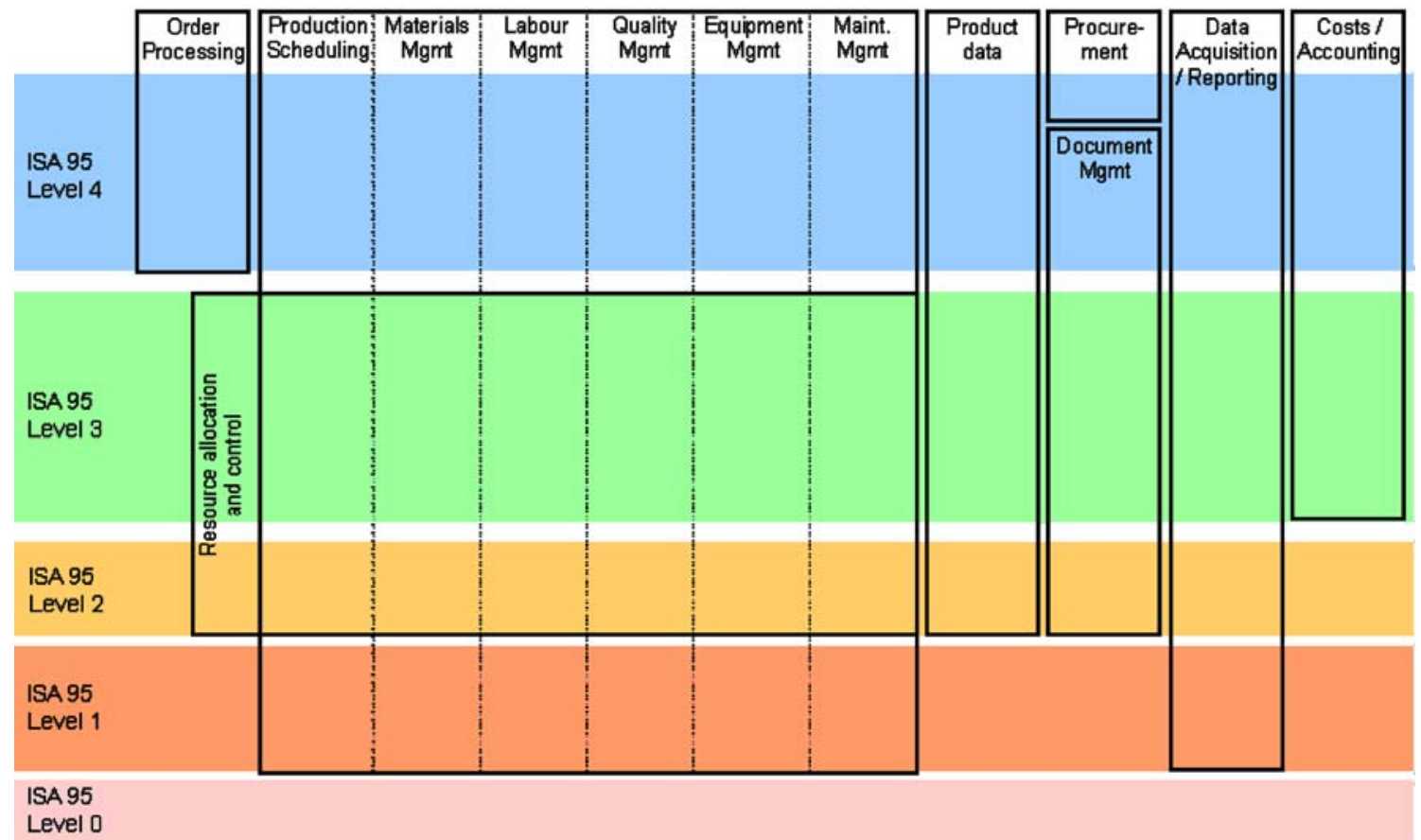

Fig. 2 The ISA 95 operating philosophy functional framework for activities (note ISA 95 levels are different from PQLI Control Strategy Levels) 
for validation based on knowledge-rich areas within the Design Space.

- Allow the plant to select optimization mode by running a particular predefined recipe that assures acceptable CQAs based on incoming material quality attributes (e.g. selecting a recipe based on particle size distribution from the previous milling step or a raw material vendor.)

- Incorporation of recipe parameter adjustments within a predefined recipe based on incoming material quality attributes.

- Implement a multivariate control model to update setpoints to operate within the design space.

- Make the Design Space knowledge available to the plant to help them utilize the new set-point freedom to achieve manufacturing objectives (e.g. reduce cycle time or increase yield)

Underpinning many of the options above will be information gained by tracking and trending of the manufacturing process on a batch-by-batch basis. Currently operators typically only have access to information about the current batch.

\section{Control Strategy}

Controls may have to handle non-contiguous operating regions. Most control systems allow one set of set-point limits on a parameter. If there were 3 interacting CPPs, how could an operator jump to a new operating region? This is a problem to consider for control system programming, and in the longer term by control system vendors.

The DS/DP manufacturing Design Space is identified during product development and measurement techniques for the CPPs are developed. Many will be based on traditional univariate measurements such as temperature, flow, and pressure but some may require multivariate measurements such as spectroscopy. In either case the proposed technology should be defined and documented.

Some multivariate techniques such as near infrared (NIR) spectroscopy may not provide a direct measurement of the process parameter of interest. In order to successfully use these techniques it is necessary to conduct an experimental programme (Design of Experiment - DOE) taking spectra and process samples; then analysing them using a reference method performed on traditional laboratory instruments e.g. Chromatography or Karl- Fisher.

Chemometric modelling can then be applied to the spectra and reference analysis data to determine if a correlation exists between the reference results and one or more cases of absorbance at specific wavelengths. These activities result in a process model that is analogous to the chemical engineering process models described earlier.

Models will need to be validated usually through the use of a set of independent measurements and samples.

Multivariate analysis techniques will need to be transformed into a practical engineering solution before being used in manufacturing. This may include risk assessments, design of sampling system, integration with the process control system, and data management. The control model includes the real time execution of the process model to produce univariate estimates that can be used by the process control system to maintain the process parameter within its acceptable working limits.

For example, a NIR spectrometer may be used to monitor material in a fluid bed dryer and the estimated water content used by the process control system to stop the drying process when the material has reached the desired moisture level.

There should be traceability from each CQA to associated CPPs and to the instruments that provides direct or indirect measurement. This information may be included in a critical instrument register so as to ensure that they are regularly tested or calibrated and maintained. These instruments should be covered during risk assessment.

The information obtained from process modelling and process equipment design can be used to define an operating alarm philosophy. For example, safety related (operator and equipment) alarms could be classified separately from product related alarms associated with CQAs and CPPs.

\section{Record Keeping and Data Management}

There are stringent requirements for regulatory record keeping. Records for regulatory compliance and product release are usually managed by the Quality Unit. The knowledge-rich enhanced pharmaceutical development approach may require much more data to be collected and managed to enable continual process improvement during the product lifecycle. This data is likely to include traditional univariate process variables such as flows or temperatures, but may also include multivariate data like particle size distributions and spectra. The alignment of time-stamps and the ability to relate various unit operations via batch ID is necessary for later data analysis.

Data can be collected in an ad-hoc manner from individual process equipment and combined with batch record data to satisfy the quality and GMP requirements, but this does not necessarily facilitate using the data for process improvement and analysis of any batch to batch variations. 
A strategic data management framework that supports both types of data may be described based on the ISA-95 standard (see Fig. 3).

\section{ISA-S95 Level 1 - Process Sensing and Manipulation}

ISA 95 Level 1 consists of traditional measurement equipment for univariate process variables (e.g. flow, temperature, or pressure) and more advanced instrumentation for the determination of process or material physical and chemical properties. The latter often result in multivariate data from instruments such as spectrometers and particle size measurement systems. These instruments may require additional supporting systems for performance monitoring and standardisation to ensure successful and reliable long-term operation. ISA 95 Level 1 also consists of actuators or devices to manipulate the process but this is outside the scope of this paper.

\section{ISA-S95 Level 2 - Monitoring and Control of the Process}

ISA 95 Level 2 systems are of particular interest as they form a bridge between analytical and process measurement systems and data management and modelling systems.

Process monitoring and control is well established and implemented using Supervisory Control and Data Acquisition (SCADA) systems together with programmable logic controllers (PLCs). Distributed Control Systems (DCS) are also used. These systems can connect to sensors from many different vendors and mainly deal with univariate process variables and simple raw data transformations. This provides visualisation of the process and allows controlled interaction with the process via the control system.

Sensor and instrument management and control should allow the setup of Process Analytical Technology (PAT)

\begin{tabular}{|c|c|c|c|c|c|}
\hline \multicolumn{5}{|c|}{$\begin{array}{c}\text { Real Time Release } \\
\text { Process Understanding }\end{array}$} & s95 \\
\hline \multicolumn{5}{|c|}{ Knowledge Management } & $\begin{array}{l}13495 \\
\text { Level } 14 \\
\end{array}$ \\
\hline \multicolumn{5}{|c|}{ PAT Data Management and Process Historian } & \multirow{2}{*}{$\begin{array}{l}\text { ISA95 } \\
\text { Level } 3\end{array}$} \\
\hline \multicolumn{5}{|c|}{ Chemometric Model Maintenance } & \\
\hline \multicolumn{2}{|c|}{$\begin{array}{l}\text { Sensor \& Instrument } \\
\text { Management \& Control }\end{array}$} & \multicolumn{3}{|c|}{ Process Monitoring \& Control } & $\begin{array}{l}\text { ISA95 } \\
\text { Level } 2\end{array}$ \\
\hline $\begin{array}{l}\text { Instruments \& } \\
\text { Simulators }\end{array}$ & \multicolumn{2}{|c|}{$\begin{array}{l}\text { Instrument } \\
\text { Performance } \\
\text { Monitoring }\end{array}$} & \multicolumn{2}{|c|}{$\begin{array}{c}\text { Instrument } \\
\text { Standardisation }\end{array}$} & $\begin{array}{l}\text { ISA95 } \\
\text { Lavel1 }\end{array}$ \\
\hline Support & Training & \multicolumn{2}{|c|}{ Strategy } & Standards & \\
\hline
\end{tabular}

Fig. 3 Data management framework based on ISA 95 standard (Note ISA 95 levels are different from PQLI Control Strategy Levels) instruments from different vendors and support the creation and execution of complex analytical methods or workflows. The workflow should allow control of the PAT instruments and use of the multivariate data obtained, together with real time chemometric prediction engines to calculate scalar prediction variables that can be used by the associated process monitoring and control system.

The management of the multivariate raw data and associated prediction variables is critical and will require metadata to describe the context of the measurement(s) made. In the simplest case this will be software provided by the vendor to operate a specific instrument. Major challenges include lack of multivariate data exchange standards, file structure standards, instrument control standards and interfacing to process monitoring and control systems.

\section{Alarm Management}

If a CPP goes outside the normal operating range, the operator should be notified and the event logged and addressed as required by the Quality System. Automatic actions may be appropriate such as putting the process on hold, shutting down the process or possibly diverting material to scrap if outside the approved Design Space. Like set point limits, alarm limits may need to take the Design Space into account. Alarm limits may need to be dynamically adjusted for individual CPPs to match the specific normal operating range. This is more complicated than static steady state limits and more similar to phase specific alarm limits for handling start-up and shutdown sequences.

Warning limits inside the normal operating ranges are also desirable. Actions on these warnings would be process and plant specific. If the process leaves the Design Space, the Quality System should define the appropriate actions.

If isolated operating regions exist, and transitions between regions are allowed during processing, alarm suppression during the transition is a consideration, as is a control plan to manage the transition and impact on product.

Outputs such as charts showing the process is operating in control within the Design Space over time are helpful for investigations and trending.

\section{ISA-S95 Level 3 - Data Management Systems and Historians}

ISA Level 3 PAT data management systems should include support for multivariate analytical method development. This in turn generates requirements for managing laborato- 


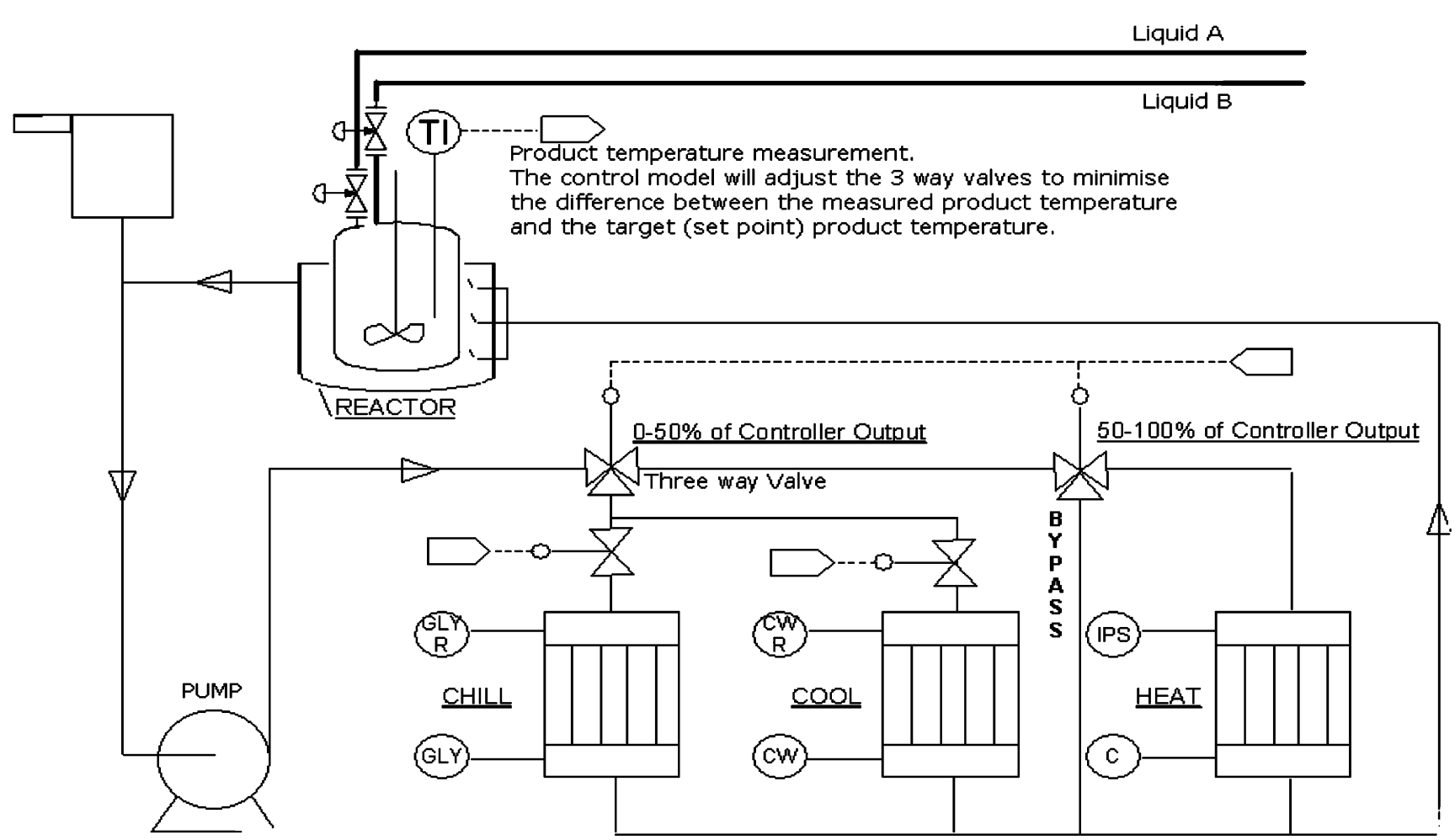

Fig. 4 Process example

ry reference data, integration with chemometric modelling software and management of models.

There is little guidance on how this data can be identified, indexed, searched or otherwise used for method development, making predictions or generating GMP related reports.

Metadata, such as the context in which a measurement has been made, is important in PAT data management. Means should therefore be provided to generate and maintain the metadata associated with the measurements.

PAT data management should not be considered in isolation. It may form part of a larger technology infrastructure that includes process control and automation. Decisions should be based on an understanding of the overall technology infrastructure for development and manufacturing.

\section{ISA-S95 Level 4 - Business Planning and Logistics}

ISA Level 4 systems are typically used for establishing the basic plant schedule, production, material use, and inventory levels.

In the context of PAT and enhanced process development, there are various possibilities in the areas of real-time release and knowledge management (a systematic approach to acquiring, analyzing, storing and disseminating information related to products, processes and components as defined by the ICH Q10 EWG). In the future this may be achieved through the use of information technology portals ${ }^{2}$.

\section{Example of Process Model and Control Model}

See also Fig. 4 above.

1. A reaction stage of a drug substance manufacturing process, requires a fixed mass of Liquid $\mathrm{A}$ to be charged into a reactor.

2. Liquid A should be heated until it reaches a steady state temperature of 30 Deg C.

3. A matching molar quantity of Liquid B should be charged into the reactor at a rate of $1 \mathrm{Kg} / \mathrm{Min}$.

4. An exothermic reaction occurs and the temperature must not exceed 40 Deg C

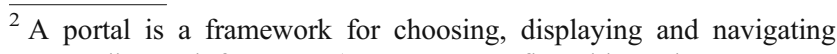
among diverse infospaces. (e.g. a user configurable Web page set up for batch release). An infospace is a collection of portlets intended to support a particular role, business process or area of knowledge, e.g. a PAT infospace. A portlet is a clearly defined collection of information, presented in a window and reusable in different contexts and views, e.g. PAT measurements associated with a specific product/formulation and batch.
} 
The process model would require knowledge of the chemical and physical properties of the liquids and thermodynamic properties of the reaction so that the heat of reaction could be calculated and the temperature rise predicted.

From this modeling, a decision on using a jacketed reactor was taken.

Further process modeling taking into account the surface area of the jacket, the heat transfer fluid used and the rate at which the heat of reaction could be removed from the reactor made it necessary to include a heat $/ \mathrm{cool} / \mathrm{chill}$ system in the plant design.

The control model defines how the requirements of the process model could be met.

Risk assessments would be applied to ensure that all control activities could take place in a safe and effective manner.

For a batch process the principles contained in the ISA S88 [5] batch control standard would be followed.

In this example the quantity of liquid B to be charged could be calculated based on the actual mass of liquid $A$ that was charged. The control model would include checks to ensure that there was sufficient capacity in the reactor to receive the charge. The control system would know which plant valves needed to be opened to establish a route from a storage tank to the reactor. During the transfer it would continuously monitor the level in the reactor to ensure it was not overfilled.

How could the reactor temperature be maintained? In one scenario, the control model would compare the actual temperature of the reactor contents with the required temperature and adjust the temperature of the heat transfer fluid to minimize the error. The temperature of the heat transfer fluid could be adjusted by diverting the fluid through chilling, cooling or heat exchangers.

\section{References}

1. ANSI/ISA-95.00.01-2000, Enterprise-Control System Integration, Part 1: Models and Terminology,

2. ANSI/ISA-95.00.02-2001, Enterprise-Control System Integration, Part 2: Object Model Attributes, contains additional details and examples to help explain and illustrate the Part 1 objects.

3. ANSI/ISA-95.00.03-2005, Enterprise-Control System Integration, Part 3: Activity Models of Manufacturing Operations Management, presents models and terminology for defining the activities of manufacturing operations management.

4. ANSI/ISA-95.00.05-2007, Enterprise-Control System Integration, Part 5: Business-to-Manufacturing Transactions, defines the transactions to interface business and manufacturing activities.

5. ANSI/ISA S88.01-1995, Batch Control Part 1: Models and Terminology

6. PQLI Control Strategy Model and Concepts DOI 10.1007/s12247008-9035-1

If you would like to provide comments to the authors regarding this paper, email feedback@ispe.org and include "PQLI Engineering Controls" in the subject line. 